\title{
Gènes et absorption intestinale des microconstituants lipidiques (vitamines liposolubles, caroténoïdes et phytostérols)*
}

Patrick BOREL

INRA, U1260 "Nutrition, Obésité

et Risque Thrombotique", Marseille, F-13385, France ;

INSERM, U1062, Marseille, F-13385,

France ;

Aix-Marseille Université, Marseille,

F-13385, France

$<$ Patrick.Borel@univ-amu.fr $>$

\begin{abstract}
Genes and intestinal absorption of lipidic microconstituents (fat-soluble vitamins, carotenoids and phytosterols)

Intestinal absorption of lipidic microconstituents $(L M)$ is very variable and depends on numerous factors. Recent studies have shown that, contrary to what was assumed, proteins are involved in absorption of $L M$ by enterocyte. It is likely that genetic polymorphisms in genes encoding for these proteins modulate absorption efficiency of these compounds. This hypothesis can explain the huge interindividual variability in absorption efficiency of these compounds. The effect of genetic variants on absorption efficiency of LM may in turn lead to modify plasma and tissues concentration of LM and, finally, their ability to prevent some diseases (cardiovascular diseases, cancers...). Recent results support these hypothesis. However, other studies are required to definitely conclude on the real effect of genetic variants of intestinal transporters of $L M$ on status and health effect of these compounds. Since most $L M$ seems beneficial for health at low (dietary) doses and can be hazardous at higher (pharmacological) doses, it can be anticipated that recommended dietary allowances of $L M$, or doses of $L M$ in functional foods/supplements, will be adapted to groups of the population carrying common genetic variants known to significantly affect $L M$ bioavailability.
\end{abstract}

Key words: genetic polymorphisms, vitamins, carotenoids, bioavailability, nutrition

pathologies (certains cancers, maladies cardiovasculaires...). Les mécanismes moléculaires de l'absorption des micronutriments et microconstituants lipidiques (ML) ont récemment été révolutionnés par la découverte que des protéines présentes dans la cellule absorptive intestinale, l'entérocyte, sont impliquées dans ce processus. Cette découverte a permis d'émettre I'hypothèse que des variations mineures dans les gènes codants pour ces protéines pourraient affecter l'efficacité d'absorption de ces composés. Des résultats récents supportent cette hypothèse et permettent $d^{\prime}$ 'imaginer que les recommandations nutritionnelles en ces composés pourraient être segmentées en fonction des caractéristiques génétiques des groupes de la population.

Les vitamines liposolubles $(A, D, E, K)$, les caroténoïdes et les phytostérols sont les principaux $\mathrm{ML}^{1}$ présents dans notre alimentation (tableau 1). II y a un net regain d'intérêt pour l'étude des mécanismes d'absorption des vitamines liposolubles car des études récentes suggèrent que, parallèlement à leurs rôles biologiques bien connus, celles-ci sont aussi impliquées dans la prévention de certaines pathologies [1-3]. II y a aussi un fort intérêt pour les

\footnotetext{
${ }^{1}$ Les microconstituants alimentaires sont des molécules organiques présentes en faible quantité dans I'alimentation $(<1 \mathrm{~g} / \mathrm{j})$. Sont qualifiés de micronutriments les microconstituants dont l'essentialité pour l'homme a été prouvée, c'est le cas des vitamines, mais ce n'est pas le cas des caroténoïdes et des phytostérols. Sont qualifiés de microconstituants lipidiques ceux qui ne sont pas solubles dans l'eau mais qui le sont dans des solvants organiques.
} risation.

Pour citer cet article : Borel P. Gènes et absorption intestinale des microconstituants lipidiques (vitamines liposolubles, caroténoïdes et phytostérols). OCL $2012 ; 19(4)$ : 209-215. doi : 10.1684/ocl.2012.0450 
Tableau 1. Les principaux microconstituants lipidiques : apports et rôles biologiques.

\begin{tabular}{|c|c|c|c|c|}
\hline Nom usuel & $\begin{array}{l}\text { Principales espèces } \\
\text { moléculaires retrouvées } \\
\text { dans I'alimentation } \\
\text { des pays industrialisés }\end{array}$ & ANC $^{a}$ & $\begin{array}{l}\text { Consommation } \\
\text { quotidienne } \\
\text { moyenne/médiane }\end{array}$ & $\begin{array}{l}\text { Principales activités } \\
\text { biologiques }\end{array}$ \\
\hline Vitamine A préformée & Rétinyl-palmitate & $900 \mu \mathrm{g}$ & $598-682 \mu \mathrm{g}$ & Activités vitaminiques $\mathrm{A}$ \\
\hline $\begin{array}{l}\text { Caroténoïdes } \\
\text { provitaminiques }\end{array}$ & $\begin{array}{l}\beta \text {-carotène } \\
\alpha \text {-carotène } \\
\beta \text {-cryptoxanthine }\end{array}$ & $\begin{array}{l}- \\
-\end{array}$ & $\begin{array}{l}2,15-2,62 \mathrm{mg} \\
0,39 \mathrm{mg} \\
0,12-0,14 \mathrm{mg}\end{array}$ & $\begin{array}{l}\text { Activités vitaminiques } \mathrm{A} \\
\text { et propriétés antioxydantes }\end{array}$ \\
\hline Vitamine $\mathrm{E}$ & $\begin{array}{l}\text { d- } \alpha \text {-tocophérol } \\
\text { d- } \gamma \text {-tocophérol }\end{array}$ & $15 \mathrm{mg}$ & $\begin{array}{l}9,8-10,3 \mathrm{mg} \\
-\end{array}$ & $\begin{array}{l}\text { Activités vitaminiques } \mathrm{E} \\
\text { et propriétés antioxydantes }\end{array}$ \\
\hline Vitamine D & Cholécalciférol (vitamine D3) & $5 \mu \mathrm{g}$ & $2,9 \mu \mathrm{g}$ & Activités vitaminiques $\mathrm{D}$ \\
\hline Vitamine $\mathrm{K}$ & $\begin{array}{l}\text { Phylloquinone } \\
\text { Ménaquinone }\end{array}$ & $120 \mu \mathrm{g}$ & $\begin{array}{l}70-80 \mu \mathrm{g} \\
21 \mu \mathrm{g}\end{array}$ & Activités vitaminiques $\mathrm{K}$ \\
\hline $\begin{array}{l}\text { Caroténoïdes non } \\
\text { provitaminiques }\end{array}$ & $\begin{array}{l}\text { Lycopène } \\
\text { Lutéine/zéaxanthine }\end{array}$ & - & $\begin{array}{l}6,6-12,7 \mathrm{mg} \\
2,0-2,3^{\mathrm{c}}\end{array}$ & Propriétés antioxydantes \\
\hline Phytostérols & $\begin{array}{l}\text { Sitostérol } \\
\text { Stigmastérol } \\
\text { Campestérol }\end{array}$ & $\begin{array}{l}- \\
- \\
-\end{array}$ & $167-437 \mathrm{mg}$ & Inhibiteurs de l'absorption du cholestérol \\
\hline
\end{tabular}

${ }^{a}$ Apports nutritionnels conseillés (ANC) pour un homme adulte. II n'existe pas d'ANC pour les caroténoïdes et les phytostérols.

${ }^{b} D^{\prime}$ un homme adulte.

${ }^{\mathrm{c}}$ Lutéine + zéaxanthine.

caroténoïdes qui sont des pigments végétaux aussi suspectés de jouer un rôle préventif dans la survenue de certains cancers et des maladies cardiovasculaires et neurodégénératives [4-7]. Par ailleurs, la lutéine et la zéaxanthine, deux caroténoïdes appartenant à la sous-classe des xanthophylles, semblent jouer un rôle important dans la fonction oculaire et pourraient prévenir la cataracte et la dégénérescence maculaire liée à l'âge $[6,8,9]$. Enfin, les stérols végétaux, ou phytostérols, sont maintenant bien connus pour leur capacité à diminuer l'absorption du cholestérol.

Des études récentes ont montré que, contrairement à ce que I'on pensait, un certain nombre de protéines présentes dans l'entérocyte sont impliquées dans l'absorption des ML. Cette découverte pourrait avoir des conséquences importantes sur les apports recommandés en ces composés à effet santé. En effet, des variations génétiques des gènes codants pour ces protéines pourraient affecter l'expression ou l'activité de celles-ci et, par conséquent, la capacité des sujets porteurs de ces variants génétiques à absorber les ML. On peut ainsi envisager que des groupes de sujets porteurs de variations génétiques défavorables sur des gènes codants pour des protéines impliquées dans l'absorption des ML pourraient nécessiter des recommanda- tions ou apports personnalisés en ces ML.

\section{Les facteurs qui affectent I'absorption des microconstituants lipidiques}

L'efficacité d'absorption des ML est très variable et dépend de nombreux facteurs [10]. Un nombre considérable de publications est consacré à l'étude de ces différents facteurs. Ces travaux ont par exemple montré que I'efficacité d'absorption de certains ML dépend :

- de la cuisson des aliments contenant les $\mathrm{ML}$;

- de la composition des aliments consommés en même temps que les sources de $\mathrm{ML}$;

- de l'activité des enzymes digestives ; - de l'efficacité des transporteurs intestinaux impliqués dans le captage des $M L$, etc.

L'acronyme Slamenghi, qui est un terme mnémotechnique proposé pour lister les facteurs qui affectent la biodisponibilité des caroténoïdes [11], peut être utilisé pour lister les facteurs qui affectent la biodisponibilité des autres ML. Chaque lettre correspond à un facteur :
- S pour species of ML (espèce moléculaire du $\mathrm{ML}$ ) ;

- L pour molecular linkage (estérification ou conjugaison de la molécule);

- A pour amount consumed in a meal (quantité de $\mathrm{ML}$ dans le repas);

- M pour matrix in which the microconstituant is incorporated (effet de la matrice alimentaire) ;

- E pour effectors of absorption (autres molécules modulant l'absorption : lipides, fibres, médicaments...);

- $\mathrm{N}$ pour nutrient status of the host (statut de l'individu en $\mathrm{ML}$ ) ;

- G pour genetic factors (facteurs génétiques) ;

- $\mathrm{H}$ pour host-related factors (facteurs liés à l'individu : âge, sexe...);

- I pour mathematical interactions (interactions entre tous ces facteurs).

Comme cela est présenté dans le tableau 2, un nombre conséquent de données est disponible pour certains de ces facteurs qui ont beaucoup intéressé les chercheurs/technologues/ industriels de l'agro-alimentaire (effet de la matrice alimentaire, effet des effecteurs d'absorption), alors que très peu de données, voire pas du tout, sont disponibles pour d'autres facteurs, souvent plus difficiles à étudier (effets génétiques, interactions entre les facteurs). De nouveaux résultats sont publiés très régulièrement et viendront, à n'en pas douter, compléter ce tableau. 
Tableau 2. Données disponibles sur les facteurs qui sont supposés affecter l'absorption des microconstituants lipidiques.

\begin{tabular}{|c|c|c|c|c|c|c|c|c|c|}
\hline Microconstituants lipidiques & $S^{a}$ & $\mathbf{L}$ & A & $\mathbf{M}$ & $\mathbf{E}$ & $\mathbf{N}$ & G & H & 1 \\
\hline Vitamine A & 0 & + & 0 & ++ & ++ & + & 0 & ++ & 0 \\
\hline Caroténoïdes provitaminiques & ++ & + & 0 & ++ & ++ & + & + & + & 0 \\
\hline Vitamine $\mathrm{E}$ & ++ & + & + & + & ++ & 0 & + & ++ & 0 \\
\hline Vitamine D & + & + & 0 & + & ++ & + & 0 & ++ & 0 \\
\hline Vitamine $\mathrm{K}$ & ++ & 0 & + & ++ & ++ & 0 & 0 & ++ & 0 \\
\hline Caroténoïdes non provitaminiques & ++ & + & 0 & ++ & ++ & 0 & + & + & 0 \\
\hline Phytostérols & + & 0 & + & + & 0 & 0 & ++ & 0 & 0 \\
\hline
\end{tabular}

++ : plus de trois publications traitent de ce facteur ; $:$ seulement quelques références (entre une et trois) ; 0 : pas de publication sur l'effet de ce facteur.

${ }^{a} L^{\prime}$ acronyme Slamenghi est un moyen mnémotechnique de se rappeler des principaux facteurs qui sont supposés affecter la biodisponibilité des microconstituants lipidiques (ML). Chaque lettre permet de se rappeler d'un facteur : $\mathrm{S}$ : species of $M L$ (espèce moléculaire du $M L$ ), $\mathrm{L}$ : molecular linkage (estérification ou conjugaison de la molécule), A : amount consumed in a meal (quantité de $\mathrm{ML}$ dans le repas), $\mathrm{M}:$ matrix in which the microconstituant is incorporated (effet de la matrice alimentaire), $\mathrm{E}$ : effectors of absorption (autres molécules modulant l'absorption : lipides, fibres, médicaments.. .), N : nutrient status of the host (statut de l'individu en ML), G : genetic factors (facteurs génétiques), $\mathrm{H}$ : host-related factors (facteurs liés à l'individu : âge, sexe.. .) et I : mathematical interactions (interactions entre tous ces facteurs).

Tableau 3. Connaissances actuelles sur les mécanismes impliqués dans l'absorption des microconstituants lipidiques.

\begin{tabular}{|lll|}
\hline $\begin{array}{l}\text { Espèce } \\
\text { moléculaire }\end{array}$ & $\begin{array}{l}\text { Efficacité } \\
\text { d'absorption (\%) }\end{array}$ & $\begin{array}{l}\text { Caractéristiques de I'absorption } \\
\text { (à doses nutritionnelles) }\end{array}$ \\
\hline Rétinyl-palmitate & $75-99$ & $\begin{array}{l}\text { Le captage entérocytaire du rétinol est un processus de diffusion facilitée } \\
\text { impliquant un transporteur (non encore identifié) }\end{array}$ \\
\hline $\begin{array}{l}\beta \text {-carotène } \\
\alpha \text {-carotène } \\
\beta \text {-cryptoxanthine }\end{array}$ & $3,5-90$ & $\begin{array}{l}\text { Le captage entérocytaire est un processus de diffusion facilitée impliquant SR-BI, } \\
\text { mais aussi probablement d'autres protéines membranaires (CD36...) }\end{array}$ \\
\hline $\begin{array}{l}\text { d- } \alpha \text {-tocophérol } \\
\text { d- } \gamma \text {-tocophérol }\end{array}$ & $10-95$ & $\begin{array}{l}\text { Le captage entérocytaire est un processus de diffusion facilitée impliquant SR-BI et NPC1L1 } \\
\text { L'efflux basolatéral est, en partie, effectué dans les HDL d'origine intestinale via un } \\
\text { transporteur énergie-dépendant : ABCA1 }\end{array}$ \\
\hline $\begin{array}{l}\text { Cholécalciférol } \\
\text { Le captage entérocytaire implique, en partie, SR-BI et NPC1L1 (données de notre } \\
\text { laboratoire non encore publiées) }\end{array}$ \\
\hline $\begin{array}{l}\text { Phylloquinone } \\
\text { Ménaquinones }\end{array}$ & $13-80$ & $\begin{array}{l}\text { Le captage des phylloquinones est médié par un transporteur énergie-dépendant } \\
\text { non encore identifié } \\
\text { Le captage des ménaquinones est supposé être passif }\end{array}$ \\
\hline $\begin{array}{l}\text { Lycopène } \\
\text { Lutéine } \\
\text { Zéaxanthine }\end{array}$ & $\begin{array}{l}0,1-1,6 \\
\text { Phytostérols }\end{array}$ & $\begin{array}{l}\text { Le captage entérocytaire est un processus de diffusion facilitée impliquant SR-BI, } \\
\text { mais aussi probablement d'autres protéines membranaires (CD36...) }\end{array}$ \\
\hline
\end{tabular}

\section{Des protéines entérocytaires sont impliquées dans l'absorption des microconstituants lipidiques}

Il a longtemps été supposé que le cholestérol était absorbé par diffusion passive, c'est-à-dire en suivant un gradient de concentration entre la lumière du tube digestif et l'intérieur de la cellule intestinale (entérocyte). Néanmoins, ce dogme a été récemment balayé quand on s'est aperçu que l'absorption du cholestérol implique plusieurs protéines présentes dans la membrane apicale de l'entérocyte : NPC1L1, SR-BI, ABCG5 et $A B C G 8$. De la même façon, il a été démontré, il y a une dizaine d'années, que la grande majorité des phytostérols nouvellement captés par l'entérocyte sont éliminés dans la lumière du tube digestif grâce à un couple de transporteurs, ABCG5 et ABCG8. En effet, quand ces transporteurs sont défaillants (mutations dans leurs gènes), cela conduit à une absorption anormalement élevée des phytostérols, aboutissant à une pathologie héréditaire : la sitostérolémie [12]. Enfin, des études récentes, effectuées en grande partie par notre laboratoire, ont aussi montré que des protéines entérocytaires, connues pour être impliquées dans l'absorption du cholestérol, sont aussi impliquées dans l'absorption des caroténoïdes et de la vitamine E. Plus précisément, nous avons été la première équipe à montrer que l'absorption d'un caroténoïde : la lutéine implique le récepteur 
"éboueur " scavenger-receptor class $B$ type I (SR-BI) [13]. Ce récepteur a par la suite été impliqué dans l'absorption $\mathrm{d}^{\prime}$ autres caroténoïdes : $\alpha$-carotène ${ }^{2}, \beta$ carotène $^{2}$ [14] et lycopène [15]. Nous avons par ailleurs aussi été la première équipe à montrer que le captage de la vitamine $E$ ( $\alpha$ et $\gamma$-tocophérol) par l'entérocyte implique aussi ce même transporteur [16], et des travaux en cours dans notre laboratoire suggèrent qu'il est aussi impliqué dans le captage de la vitamine D3 (cholécalciférol). Ce récepteur n'est néanmoins pas " universel " puisqu'il n'est pas impliqué dans le captage du rétinol ${ }^{2}$. Cette forme de vitamine $A$ d'origine animale semble en effet être captée par un transporteur spécifique qui n'a pas encore été identifié, mais qui pourrait être STRA6 [17]. Une autre protéine de la famille des récepteurs "éboueurs » pourrait aussi être impliquée dans le captage des caroténoïdes au niveau du pôle apical de l'entérocyte, il s'agit de CD36, mais les résultats sur l'implication de cette protéine dans l'absorption des caroténoïdes sont encore contradictoires [14, 18]. Enfin, une équipe japonaise vient récemment de montrer que NPC1L1, qui ferait la navette entre la membrane apicale et le cytosol, est impliqué dans I'absorption de la vitamine E [19].

Les ML captés par l'entérocyte, et qui n'ont pas été rééfflués vers la lumière intestinale (cas des phytostérols et, peut-être, d'une partie de la vitamine $E$ [16]), doivent être transportés dans l'environnement aqueux du cytosol pour être incorporés dans les chylomicrons ou, comme certains résultats récents le suggèrent, être sécrétés dans d'autres véhicules (HDL, protéines plasmatiques) au pôle basolatéral de I'entérocyte. Du fait de leur hydrophobicité, il est probable que ces molécules sont transportées par des protéines intracellulaires. On n'a pas encore identifié toutes ces protéines, mais des protéines impliquées dans le transport intracellulaire du rétinol dans les cellules intestinales (CRBP II) et de la vitamine $E$ dans divers types cellulaires (alpha-TTP, TAP et trois sec14 like-proteins) ont été décrites.

\footnotetext{
${ }^{2}$ Borel P, Moussa M, Reboul E. SR-BI is involved in provitamin A carotenoid absorption, and genetic variation in SCARB1 is associated with blood concentrations of carotenoids in different populations. 2009 [soumis pour publication].
}

Les ML sont supposés être incorporés dans les chylomicrons qui vont les transporter vers le foie où ils seront, soit éliminés dans la bile, soit stockés (cellules étoilées du foie pour la vitamine $A$ ), soit résécrétés associés à des protéines spécifiques (RBP2 pour le rétinol, DBP pour la vitamine $D$ ), ou dans les VLDL, pour être distribués vers les tissus périphériques. L'étape initiale de ce schéma (incorporation exclusive dans les chylomicrons) commence à être remise en cause puisque des travaux de notre équipe et d'un autre laboratoire ont montré récemment qu'une partie de la vitamine E est sécrétée dans les HDL d'origine intestinale via un transporteur membranaire : ABCA1 [20, 21].

En conclusion, de nombreuses protéines, dont beaucoup non encore identifiées, sont impliquées dans le captage, le transport intracellulaire et I'efflux des ML par l'entérocyte. Le tableau 3 et la figure 1 présentent un résumé des connaissances actuelles qui ne vont pas manquer d'évoluer significativement dans les prochaines années, vu l'intérêt porté à cette thématique.

\section{Conséquences physiologiques et physiopathologiques de I'implication de protéines dans I'absorption des microconstituants lipidiques}

Le fait que des protéines soient impliquées dans l'absorption des ML permet de supposer que des facteurs génétiques puissent affecter les concentrations sanguines et tissulaires, et l'effet préven-

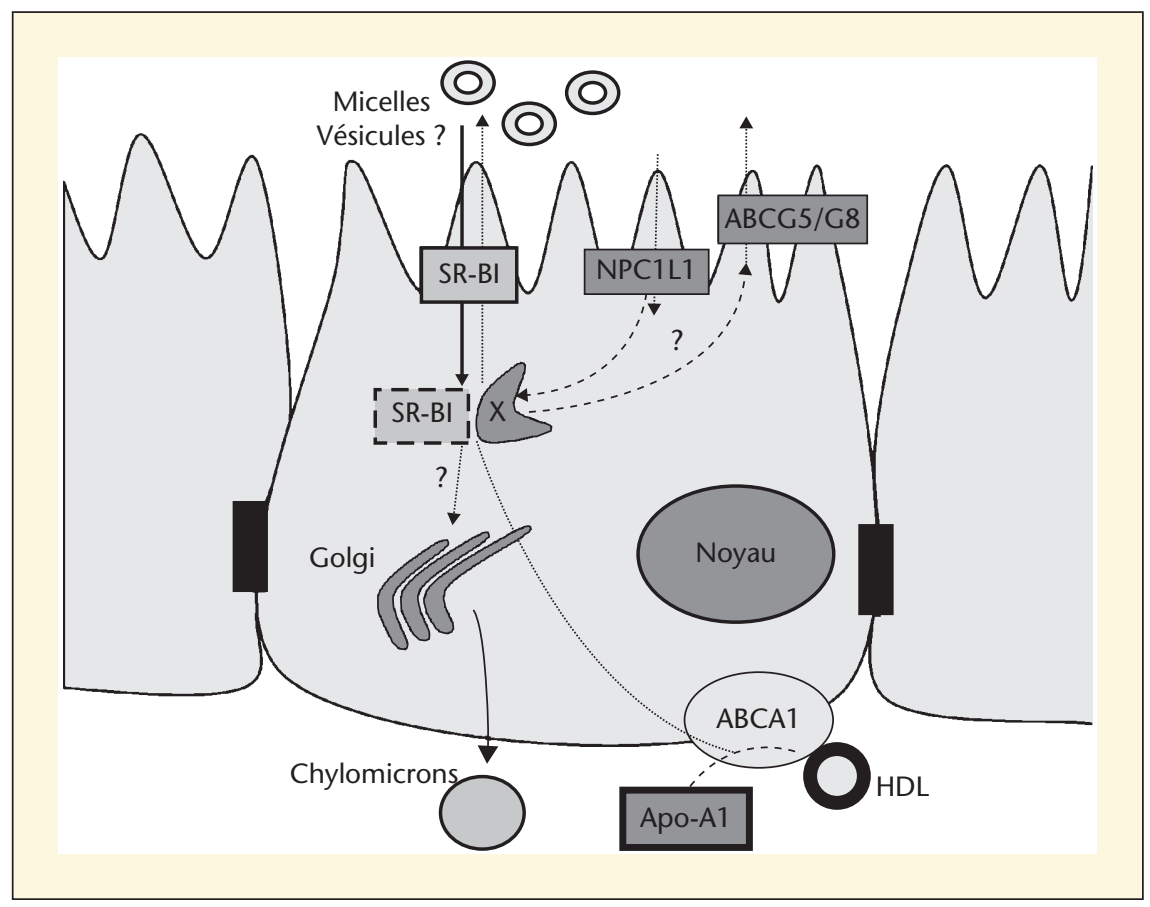

Figure 1. Connaissances actuelles sur le transport des microconstituants lipidiques (ML) dans l'entérocyte. Les $M L$ sont apportés au niveau de la membrane apicale de l'entérocyte dans des micelles mixtes et/ou des vésicules (liposomes). Le scavenger-receptor class-B type I (SR-BI) est impliqué dans le captage des caroténoïdes, des vitamines $E$ et $D$, et des phytostérols. NPC1L1 est impliqué dans le captage des phytostérols et des vitamines $D$ et $E$, mais il n'est apparemment pas impliqué dans celui des caroténoïdes. D'autres protéines présentes dans la membrane apicale pourraient également être impliquées, c'est le cas de CD36. Certains travaux suggèrent que certaines de ces protéines sont internalisées puis sont recyclées vers la membrane apicale. Après captage, certains $M L$ sont rééflués au niveau de la membrane apicale par des protéines plus ou moins spécifiques de l'efflux. Ainsi, I'hétérodimère ABCG5/G8 est responsable de l'efflux de la grande majorité des phytostérols, et SR-BI est impliqué dans I'efflux de la vitamine $E$. Les $M L$ non rééflués sont probablement transportés dans l'entérocyte par une ou des protéines non encore identifiées $(X)$. La grande majorité des $M L$ est incorporée dans les chylomicrons mais une partie est sécrétée dans les HDL d'origine intestinale via ABCA1 (cas de la vitamine $E$ ). 
tif de ces composés vis-à-vis de pathologies. En effet, il est raisonnable de penser que des polymorphismes génétiques de gènes codants pour ces protéines, les plus fréquents étant les variations d'une paire de base appelées SNP pour " single nucleotide polymorphisms ", pourraient affecter l'expression ou l'activité de ces protéines, et donc l'efficacité de l'absorption de ces molécules. Cette hypothèse est expliquée dans la figure 2 qui présente trois transporteurs putatifs d'un ML. T1 : un transporteur apical (ex. : SR-BI), T2 : un transporteur intracellulaire (ex. : L-FABP), T3 : un transporteur basolatéral (ex. : $A B C A 1)$. Des modifications d'expression ou d'activité, d'un ou de plusieurs de ces transporteurs pourraient expliquer la très forte variabilité interindividuelle de réponse aux $\mathrm{ML}$ (figure 3) et I'allure gaussienne de la distribution de réponse d'une population (figure 4) [22]. L'effet des variants génétiques sur l'absorption des $\mathrm{ML}$ pourrait par conséquence affecter les concentrations sanguines en ces composés et, in fine, les concentrations tissulaires. Ces hypothèses en cascade semblent vérifiées par des résultats récents de notre laboratoire. Nous avons en effet montré que les concentrations sanguines en caroténoïdes et en vitamine $\mathrm{E}$ sont associées à des variants génétiques de SCARB1 (le gène codant pour SR-BI) [23]. Nous venons aussi de montrer que les fréquences alléliques de deux variants de ce même gène sont linéairement corrélées aux concentrations sanguines en caroténoïdes provitaminiques ( $\alpha$-carotène, $\beta$-carotène et $\beta$-cryptoxanthine) dans différentes populations. Concernant le rôle potentiel des variants génétiques sur les concentrations tissulaires en $\mathrm{ML}$, une étude récente de notre laboratoire montre qu'un variant génétique de CD36, un récepteur potentiellement impliqué dans l'absorption des caroténoïdes, est associé à la densité optique de pigment maculaire ${ }^{3}$, valeur qui est dépendante de la concentration en caroténoïdes dans cette région centrale de la rétine. Dans la mesure où certains

\footnotetext{
${ }^{3}$ Borel P, Szabo De Edelenyi F, VincentBaudry S, et al. Genetic variants in BCMO1 and CD36 are associated with plasma lutein concentrations and macular pigment optical density in humans. 2009 [soumis pour publication].
}



Figure 2. Hypothèse permettant d'expliquer la très forte variabilité interindividuelle d'absorption des microconstituants lipidiques (ML). Cette hypothèse suppose que des variants génétiques modifient l'activité ou l'expression des protéines impliquées dans le transport des ML au sein de l'entérocyte. T1: transporteur apical putatif (ex.: SR-BI). T2: transporteur intracellulaire putatif (ex. : L-FABP). T3 : transporteur basolateral putatif (ex. : ABCA1). Un transporteur de couleur blanche signifie qu'il est correctement exprimé et que son efficacite est optimale. Un transporteur de couleur grise signifie qu'un variant génétique a partiellement diminué son expression ou son efficacité. Un transporteur de couleur noire signifie qu'un variant génétique a très fortement affecté son expression ou son activité. On suppose que, quand tous les transporteurs sont correctement exprimés et efficaces, les sujets sont "bon répondeurs " aux $M L$ transportés par ces transporteurs. Inversement, quand un ou plusieurs transporteurs voient leur expression ou leur activité profondément affectée par des variants génétiques, les sujets sont "faibles répondeurs". Enfin, quand certains transporteurs sont moins bien exprimés ou moins efficaces, alors que d'autres sont parfaitement exprimés et efficaces, les sujets sont des "répondeurs intermédiaires". Cette hypothèse pourrait expliquer les résultats présentés dans les figures 3 et 4 .

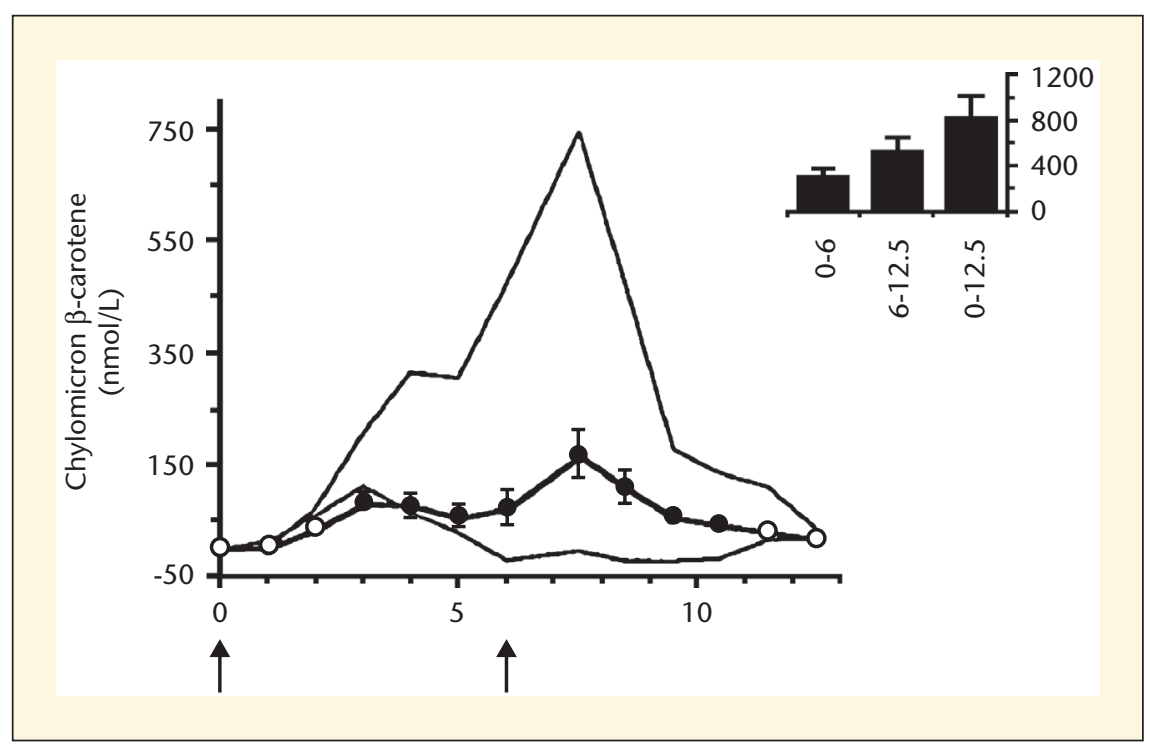

Figure 3. Variabilité interindividuelle de l'efficacité d'absorption des microconstituants lipidiques $(M L)$, exemple du $\beta$-carotène. Ce graphe représente la variation de concentration du $\beta$-carotene dans les chylomicrons en période post-prandiale suite à l'ingestion d'un repas-test apportant une dose de $120 \mathrm{mg}$ de $\beta$-carotène. Les flèches représentent les temps auxquels ont été donnés les repas-tests. Le premier apportait le $\beta$-carotène. Le second n'apportait pas de $\beta$-carotène. Le graphe avec les histogrammes représente les aires sous la courbe mesurées entre les différents temps post-prandiaux. La courbe en gras représente la moyenne des concentrations mesurées chez 16 sujets sains. La courbe la plus haute représente la réponse du sujet qui a répondu le plus (aire sous la courbe la plus importante), la courbe la plus basse celle du sujet qui a répondu le moins. Cette figure est extraite d'un article publié dans J Lipid Research en 1998 [22]. 


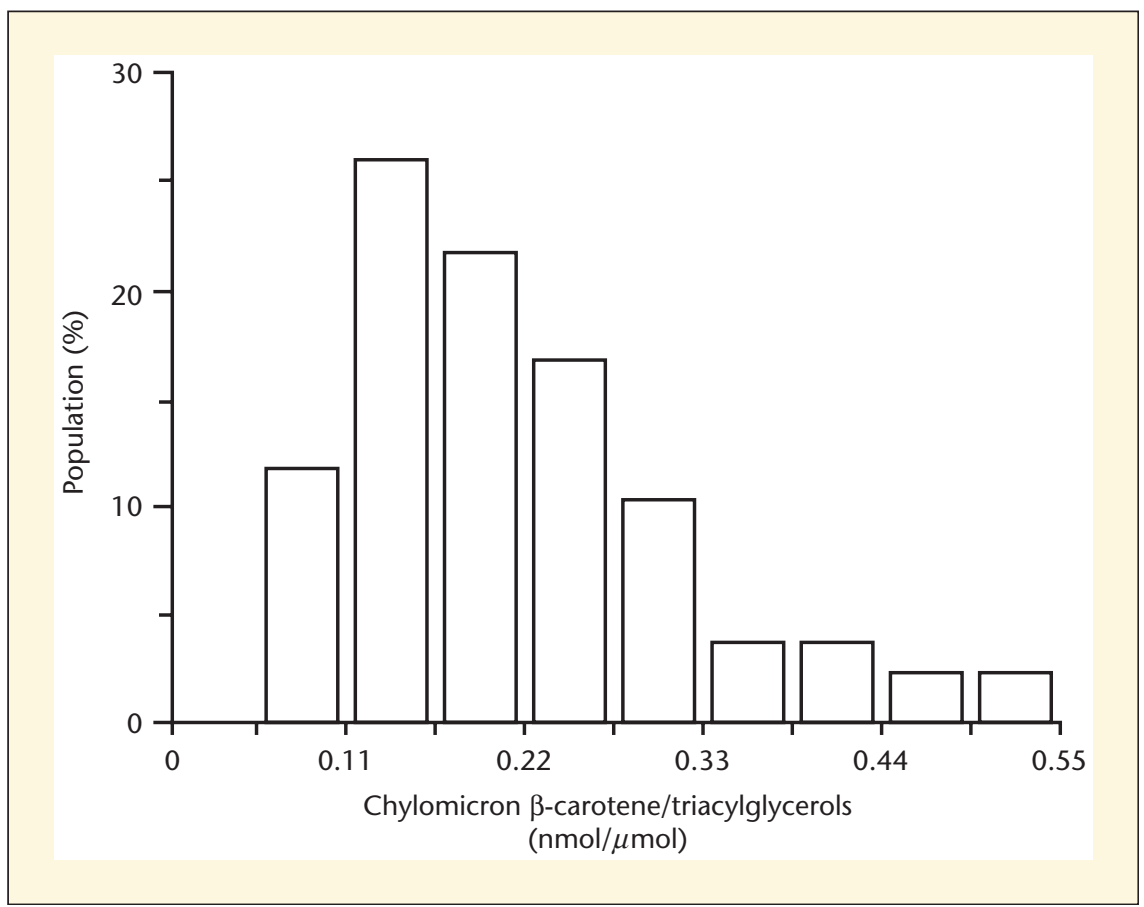

Figure 4. Variabilité de l'efficacité d'absorption du $\beta$-carotène dans une population. Ce graphe représente la distribution de la réponse post-prandiale en $\beta$-carotène dans les chylomicrons chez 79 sujets sains. La réponse était estimée en mesurant la concentration de $\beta$-carotène dans les chylomicrons trois heures après l'ingestion d'un repas-test apportant une dose de $120 \mathrm{mg}$ de $\beta$-carotène. La concentration en $\beta$-carotène était corrigée de la concentration en triglycérides des chylomicrons pour s'affranchir de la variabilité de réponse en ces lipoprotéines qui transportent le $\beta$-carotène nouvellement absorbé en période post-prandiale [22].

ML sont associés à l'incidence de certaines pathologies, on suppose que des variations dans la teneur tissulaire en ces composés pourraient affecter l'incidence de ces pathologies. C'est ce que semble suggérer les résultats d'une étude récente qui montre que la fréquence allélique d'un variant génétique dans SCARB1, gène codant pour SR-BI qui est impliqué dans l'absorption [24] et le captage de la lutéine par la rétine [25], est associée avec le risque de développer une dégénérescence maculaire liée à l'âge (résultats communiqués par le $\mathrm{Dr} \mathrm{E}$. Souied, Eye Clinic à Créteil, et non encore publiés).

\section{Application potentielle de l'effet des variants génétiques sur l'absorption des microconstituants lipidiques : la nutrition personnalisée}

Le fait que des variations génétiques puissent moduler l'absorption des ML tants en ce $\mathrm{ML}$ (sous réserve que ces apports soient inférieurs à la limite de toxicité de ces nutriments). L'effet des variants génétiques sur la prescription de certains médicaments est déjà utilisé par la pharmacogénétique. On s'est en effet aperçu que la réponse à certains médicaments (concentration sanguine notamment) est en partie dépendante de polymorphismes génétiques de gènes codants pour des enzymes de détoxification des xénobiotiques (cytochromes P450, glutathion transférases...). Par exemple, des sujets porteurs du variant CYP2D6*3/*4 sont de mauvais cataboliseurs du métropolol, un bêtabloquant utilisé dans le traitement de la tension artérielle, ou de la fluoxétine (Prozac), un antidépresseur [26]. Les posologies de ces médicaments peuvent donc être ajustées si on connaît les allèles des patients pour ces variants génétiques. On peut penser que la même approche sera utilisée en nutrition, et plus particulièrement pour les $M L$ qui peuvent être bénéfiques à doses nutritionnelles et néfastes à doses pharmacologiques [27]. On peut donc anticiper que, quand suffisamment de données seront disponibles, les ANC en certains nutriments et microconstituants seront segmentés en fonction de caractéristiques génétiques des individus, ou plus probablement de groupes d'individus. Par ailleurs, si la demande socioéconomique est là, on peut imaginer que des fabricants de suppléments alimentaires pourront proposer des formulations adaptées à des sujets porteurs de variants génétiques nécessitant des doses accrues de molécules à effet santé, du fait de leur faible capacité à les absorber (posologies pour "faibles répondeurs" et pour "forts répondeurs"). plus de 1,5 millions de personnes pour la population française !). Il y a de bonnes raisons de penser que ces sujets sont porteurs de variants génétiques induisant des modifications suffisamment importantes de leur métabolisme (absorption peu efficace, élimination trop efficace...) pour entraîner des besoins plus importants en certains (micro)nutriments. Aussi, en cas de déficience manifeste en un ML (évaluée par des dosages sanguins par exemple) et malgré des apports alimentaires apparemment suffisant en ce ML (évalués par une enquête alimentaire), on pourrait envisager de génotyper ces sujets sur des variants génétiques clés de façon à leur recommander des apports plus impor-

\section{Conclusion}

La découverte que des protéines entérocytaires sont impliquées dans I'absorption des ML est une découverte majeure dans le domaine de la biodisponibilité de ces composés. Les variations interindividuelles d'efficacité d'absorption de ces molécules peuvent être attribuées en partie à des variations génétiques dans les gènes codants pour ces protéines. Cela pourrait avoir des répercutions importantes vis-à-vis du rôle bénéfique de certains ML dans la prévention de certaines pathologies. On peut supposer que les résultats de 
nutrigénétique pourraient conduire à des recommandations plus personnalisées en ces composés, valeurs qui prendraient en compte des caractéristiques génétiques de certains groupes de la population. Bien évidemment, de nombreuses études sont encore nécessaires pour valider les relations entre certains variants génétiques et les besoins en $\mathrm{ML}$, et les recommandations en fonction de critères génétiques devront aussi satisfaire à des critères éthiques et économiques.

Conflits d'intérêts : L'auteur déclare n'avoir aucun conflit d'intérêts.

Remerciements. L'auteur remercie S. et B. Borel pour leur relecture critique et constructive du manuscrit.

\section{RÉFÉRENCES}

1. Booth SL, Broe KE, Gagnon DR, et al. Vitamin $\mathrm{K}$ intake and bone mineral density in women and men. Am J Clin Nutr 2003; 77 : 512-6.

2. Holick MF. Vitamin D: important for prevention of osteoporosis, cardiovascular heart disease, type 1 diabetes, autoimmune diseases, and some cancers. South Med / 2005 ; $98: 1024-7$.

3. Traber MG. Heart disease and singlevitamin supplementation. Am J Clin Nutr 2007 ; 85 : 293S-9S.

4. Jimenezjimenez FJ, Molina JA, Fernandezcalle $P$, et al. Serum levels of beta-carotene and other carotenoids in Parkinson's disease. Neurosci Lett 1993 ; 157 : 103-6.

5. Rao AV, Agarwal S. Role of lycopene as antioxidant carotenoid in the prevention of chronic diseases : a review. Nutr Res 1999 ; $19: 305-23$.

6. Gale CR, Hall NF, Phillips DI, Martyn CN. Lutein and zeaxanthin status and risk of agerelated macular degeneration. Invest Ophthalmol Vis Sci 2003 ; 44 : 2461-5.

7. Osganian SK, Stampfer MJ, Rimm E, Spiegelman D, Manson JE, Willett WC.
Dietary carotenoids and risk of coronary artery disease in women. Am J Clin Nutr $2003 ; 77: 1390-9$.

8. Stahl W. Macular carotenoids: lutein and zeaxanthin. Dev Ophthalmol 2005 ; 38 : 7088.

9. Richer S, Stiles W, Statkute L, et al. Doublemasked, placebo-controlled, randomized trial of lutein and antioxidant supplementation in the intervention of atrophic agerelated macular degeneration: the Veterans LAST study (Lutein Antioxidant Supplementation Trial). Optometry 2004 ; 75 : 216-30.

10. Borel P. Factors affecting intestinal absorption of highly lipophilic food microconstituents (fat-soluble vitamins, carotenoids and phytosterols). Clin Chem Lab Med 2003 ; 41 : 979-94.

11. West CE, Castenmiller JJJM. Quantification of the "SLAME-NGHI" factors for carotenoid bioavailability and bioconversion. Int J Vitam Nutr Res 1998 ; 68 : 371-7.

12. Berge KE, Tian H, Graf GA, et al. Accumulation of dietary cholesterol in sitosterolemia caused by mutations in adjacent $A B C$ transporters. Science $2000 ; 290$ : 1771-5.

13. Reboul E, Abou L, Mikail C, et al. Lutein is apparently absorbed by a carrier-mediated transport process in Caco-2 cells. Clin Nutr $2003 ; 22$ : S103.

14. van Bennekum A, Werder M, Thuahnai $\mathrm{ST}$, et al. Class B scavenger receptor-mediated intestinal absorption of dietary betacarotene and cholesterol. Biochemistry 2005 ; $44:$ 4517-25.

15. Moussa M, Landrier JF, Reboul E, et al. Lycopene absorption in human intestinal cells and in mice involves scavenger receptor class B type I but not Niemann-pick C1like 1. J Nutr 2008 ; 138 : 1432-6.

16. Reboul E, Klein A, Bietrix F, et al. Scavenger receptor class $B$ type I (SR-BI) is involved in vitamin $\mathrm{E}$ transport across the enterocyte. J Biol Chem 2006 ; 281 : 4739-45.

17. Kawaguchi R, Yu J, Honda J, et al. A membrane receptor for retinol binding protein mediates cellular uptake of vitamin A. Science $2007 ; 315: 820-5$.
18. During A, Dawson HD, Harrison EH. Carotenoid transport is decreased and expression of the lipid transporters SR-BI, NPC1L1, and ABCA1 is downregulated in Caco-2 cells treated with ezetimibe. I Nutr $2005 ; 135: 2305-12$.

19. Narushima K, Takada T, Yamanashi $Y$, Suzuki H. Niemann-pick C1-like 1 mediates alpha-tocopherol transport. Mol Pharmacol 2008 ; 74 : 42-9.

20. Anwar K, Kayden HJ, Hussain MM. Transport of vitamin $\mathrm{E}$ by differentiated Caco-2 cells. J Lipid Res 2006 ; 47 : 1261 73.

21. Reboul E, Trompier D, Moussa M, et al. ATP-binding cassette transporter Al (ABCA1) is significantly involved in intestinal absorption of alpha- and gamma-tocopherol but not in that of retinyl palmitate. Am J Clin Nutr 2009 ; $89: 1-8$.

22. Borel P, Grolier P, Mekki N, et al. Low and high responders to pharmacological doses of beta-carotene : proportion in the population, mechanisms involved and consequences on beta-carotene metabolism. J Lipid Res 1998 ; 39 : 2250-60.

23. Borel P, Moussa M, Reboul E, et al. Human plasma levels of vitamin $E$ and carotenoids are associated with genetic polymorphisms in genes involved in lipid metabolism. J Nutr 2007 ; 137 : 2653-9.

24. Reboul E, Abou L, Mikail C, et al. Lutein transport by Caco-2 TC-7 cells occurs partly by a facilitated process involving the scavenger receptor class B type I (SR-BI). Biochem J 2005 ; 387 : 455-61.

25. During A, Doraiswamy S, Harrison EH. Xanthophylls are preferentially taken up compared with beta-carotene by retinal cells via a SRBI-dependent mechanism. / Lipid Res $2008 ; 49$ : 1715-24.

26. Terra SG, Pauly DF, Lee CR, et al. Betaadrenergic receptor polymorphisms and responses during titration of metoprolol controlled release/extended release in heart failure. Clin Pharmacol Ther 2005 ; 77 : 127 37.

27. Pryor WA, Stahl W, Rock CL. Betacarotene: from biochemistry to clinical trials. Nutr Rev $2000 ; 58$ : 39-53. 